\title{
PODEX - PhOtometric Data EXtractor
}

\author{
T. Kallinger \\ Institut für Astronomie, Türkenschanzstr. 17, 1180 Vienna, Austria
}

\begin{abstract}
New reduction tools are required to handle the increasing amount of photometric CCD data within a reasonable time. PODEX is an IDL based tool developed to optimize the time needed for extracting light curves out of photometric CCD data. The GUI based semi-automatic reduction tool provides the possibility to apply bias correction, flat fielding, and background modeling to the raw data. After extracting magnitudes even in crowded fields, a color-dependent extinction correction and a conversion to relative magnitudes is performed. The output of PODEX are completely reduced light curves of an arbitrary number of stars on the CCD images. As the conditions between various nights and observing runs change continuously and sometimes dramatically, full interactivity throughout the entire reduction procedure via a powerful graphical user interface is a big advantage over all black-box procedures known to us.
\end{abstract}

\section{Introduction}

The motivation for developing PODEX originates in the increasing amount of photometric CCD data and in the need to reduce them as fast as possible and with minimum effort. Therefore, a tool is needed providing all needed reduction steps at once.

All black-box procedures known to us (like IRAF or MOMF) are complicated to handle and provide only the extraction of instrumental magnitudes. For a complete reduction also a color dependent extinction correction and the conversion to relative magnitudes is needed. PODEX provides it.

The PODEX source code can be found at

$$
\text { http://ams.astro.univie.ac.at/computer.php }
$$




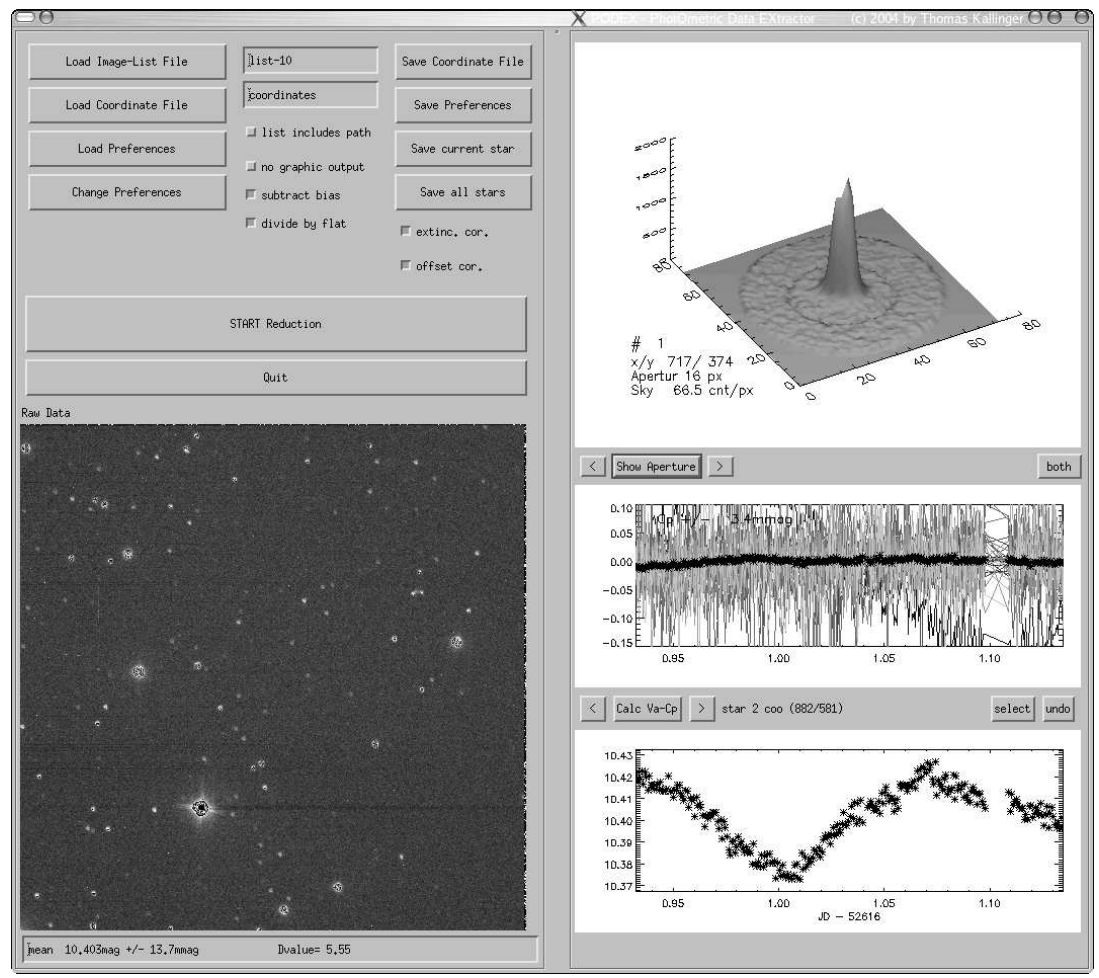

Figure 1: Graphical user interface of PODEX. top left: control field; middle left: The actual image is displayed in the raw data field. The stars of interest are marked with cross-hair symbols on the first image. bottom left: status line; top right: The apertures and background annulus of all used stars can be displayed in the aperture field to control the proper definition of the aperture radii. middle right: The greyscaled lines indicate the instrumental light curves of all used stars. The black symbols represent the corresponding reference light curve. bottom right: The relative (or absolute) light curve of the actual star is displayed in the light curve field.

\section{Input}

After starting IDL, type "podex" to start the graphical interface of PODEX. The main input for PODEX is a file containing the list of CCD images to reduce (in FITS format). PODEX is able to handle compressed FITS images as well. If the images are not stored in the same directory as the list file (because the data are on a CD or DVD, e.g.) define the full path in the list file and use the list includes path option in the main window. 


\subsection{Preferences}

The preferences of the actual session are controlled via the preference menu (click the Change Preferences button in the control field). There is also the possibility to import a set of preferences from a file (Load Preferences). The session preferences are:

- FITS header keywords for date, Universal Time and integration time of the observations:

Date and time are needed for calculation of the Julian date. Intensities are normalized to ADU per, i. e. dividing by integration time.

- Right ascension (h:m:s) and declination (d:m:s) of the image center:

Stellar coordinates at epoch 2000.0 are used for the heliocentric correction of the Julian date and the determination of the zenith distance.

- Geographic latitude (d:m:s) and east longitude (h:m:s) of the observatory:

Geographic coordinates are used for calculation of the zenith distance needed for the photometric extinction correction.

- Filename of bias and flat field image:

Bias correction and/or flat fielding can be activated by using the subtract bias and/or divide by flat options in the main window.

- Center box (pixel) for image centering:

Size of the box in which PODEX searches for the center of the star used to determine the telescope pointing offset of consecutive images.

- Radius of the aperture (pixel):

Aperture radius for the brightest used star on the image.

- Inner radius and width (pixel) of the background annulus.

- Scaling factor for individual apertures (pixel per mag):

The aperture radius for fainter stars decreases by the given scale factor.

- Magnitude of star \#1:

In order to compensate transparency changes in consecutive nights, the magnitude of star \#1 is used as a reference.

When using a preference file, the first line of the file indicates the actual working path. Click Save Preferences to save the actual preferences.

\subsection{Coordinates}

The pixel coordinates of the stars of interest are imported from a three column file. The first two columns contain the $X$ - and $Y$-coordinates on the image. The third column an arbitrary color index (Johnson B-V, e.g.) for the corresponding star. The color index is used for the color-dependent extinction correction. If no color index is known, the value should be zero. 
The first star (star \#1) in the list plays a special part. This star is used for determining coordinate offsets of consecutive images due to an insufficient pointing stability of the telescope. The star is also used as a reference light source (use the offset correction option in the main window) to calibrate relative magnitudes to absolute magnitudes and to compensate transparency changes in consecutive nights. Therefore this star has to meet some special conditions.

- Star \#1 should be a bright and constant star.

- Star \#1 should not be located near the borders of the CCD frame.

- Inside the defined box for image centering no brighter star should be present.

PODEX provides the possibility to create a list of pixel coordinates. When moving the cursor onto a target (in the raw data field) and clicking the left mouse button, the center of the star is determined and marked by a cross-hair symbol. Click the right mouse button, and the actual $X / Y$ position of the cursor and the corresponding intensity (in ADU) are displayed in the status line. Using the Save Coordinate File button, the produced list of pixel coordinates (with color index equal to zero) is exported to a file.

\section{What is going on?}

The reduction process is started by clicking the START Reduction button. First, the bias image is subtracted from the raw image and the residual image is divided by the flat field image (optional). After these corrections the image is divided by integration time to normalize the intensity to ADU per second. A sub-image (the size is defined by the preference parameter center box) centered on the pixel coordinates of star \#1 is extracted from the image. The difference between the given coordinates for star \#1 and the center of a 2-D Gaussian fit to the sub-image defines the pointing offset in $X$ and $Y$ direction of the actual image. This offset is applied to all coordinates.

\subsection{The first image}

The first image is used to define the individual aperture radii for all stars listed in the coordinate list. As illustrated in Fig. 1 and Fig. 2, there is a clear relation between the brightness of a star and the optimum aperture size (determined by minimization of the point-to-point scatter in the light curve). Following increasing concentric circles from the center of a star's PSF to the border, the signal-to-background scatter ratio grows smaller. When this ratio falls below a certain level, including further (less exposed) pixels decreases the signal to noise ratio of the resulting light curve. The turning point, where including pixels at the edge of the PSF starts to decrease the resulting quality, is reached earlier for faint stars than for bright stars. 
For all stars listed in the coordinate file, the total amount of ADUs inside the default aperture (defined by the preference keyword aperture radius) gives the stellar intensity. The mean background intensity is subtracted from the stellar intensity (see next section), and the residuals are transformed into instrumental magnitudes. The instrumental magnitudes are used to define the individual aperture radii. If this option is not needed, set the preference keyword scale factor to zero. In this case the default aperture radius is used for all stars. Instrumental magnitudes and the corresponding aperture radii are listed in the terminal window. This list can be used to acquire color indices when starting with images obtained with different filters.

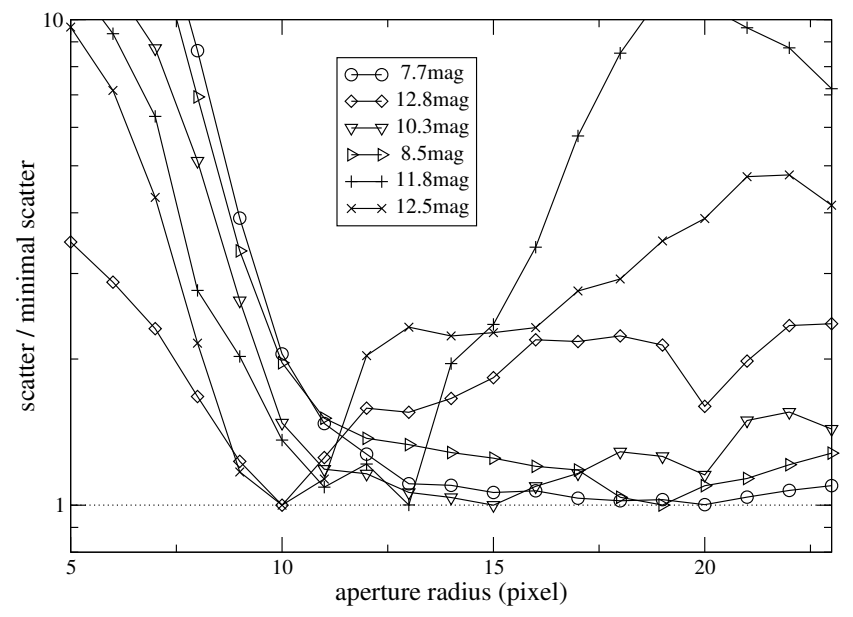

Figure 2: Point-to-point scatter versus aperture radius for stars with different magnitudes. The scatter decreases with increasing aperture radius, reaches a minimum, and starts to increase again. The size of the aperture with the least scatter in the light curve depends on the brightness of the star. For fainter stars, smaller apertures yield to optimum photometric quality.

\subsection{Background correction}

To use an annulus around the aperture to determine the background level is sometimes problematic in crowded fields when one or more stars are located in the background annulus. Nevertheless, PODEX uses such a background annulus 


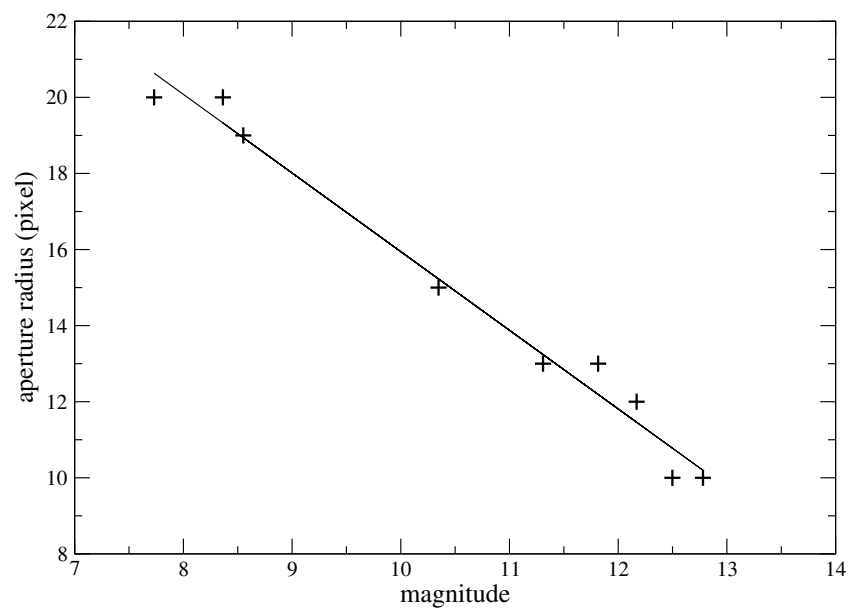

Figure 3: Mean instrumental magnitude versus aperture radius associated to minimum point-to-point scatter in the resulting light curve. The solid line represents a linear regression, illustrating the high degree of linearity in the relation between aperture radius and stellar brightness. Here the individual aperture radius decreases with about 2 pixel per magnitude.

but tries to ignore pixels exposed to stellar light (or cosmics). Hence the mean intensity level and standard deviation of all stars inside the background annulus (defined by the preference keywords inner radius and width) is determined. If a pixel intensity exceeds the mean level by more than three times the standard deviation, the corresponding pixel is rejected. The mean intensity and standard deviation of all accepted pixels is re-calculated. The procedure is repeated until no pixels are rejected but only up to 5 times.

\subsection{Extraction of instrumental light curves}

After defining the individual aperture radii, the reduction procedure extracts the total intensity for all apertures, subtracts the corresponding mean background level (multiplied by the number of pixels inside the aperture), and transforms the residuals into instrumental magnitudes for all images given in the input file. 

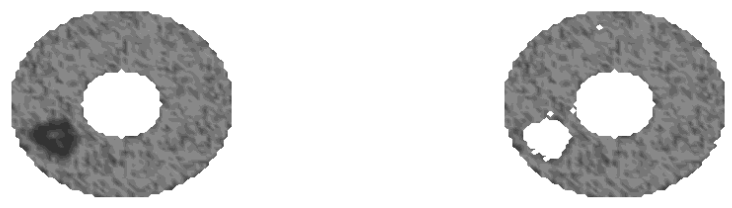

Figure 4: Left: Background annulus with containing a star. Right: Pixels exposed to stellar light are not used to determine the mean background level around the aperture (the star of interest is located inside the inner circle).

\subsection{Color-dependent extinction correction}

If a color index $(\mathrm{Cl})$ is given in the coordinate file and the option extinc. cor. in the control field is selected a color dependent extinction correction is performed for all instrumental light curves. First, the mean magnitude is subtracted from all instrumental light curves. Then the extinction coefficient for an individual light curve is computed as the slope of a linear regression in the Bouguer plot (magnitude versus airmass $\mathrm{X}$ ). There is a linear relation between the extinction coefficients and the corresponding $\mathrm{Cl}$. Again, a linear regression is used to define the coefficients $k_{0}$ and $k_{1}$. The instrumental light curves are corrected by

$$
\operatorname{mag}_{\text {corr }}=\text { mag }_{\text {instrumental }}-X \cdot\left(k_{0}+C I \cdot k_{1}\right) .
$$

If no color indices are given, the extinction correction is not necessary because the differential extinction for different stars is too small to effect the resulting light curves. (The typical field of view of a CCD images is not larger than a few arcminutes). The average extinction of the field of view is corrected by subtracting the reference light curve.

\subsection{Reference light curve}

The reference light curve corresponds to the weighted mean of all instrumental light curves. The weight of an individual light curve is defined by the inverse D-value divided by the variance of the corresponding light curve. The D-value is the ratio between the standard deviation and the point-to-point scatter of a light curve. The D-value is used as an estimator for variability in the light curve. A light curve with a high D-value and/or a high variance has low weight 
for the reference light curve. Clicking the $\mathrm{Calc} V \mathrm{Va}-\mathrm{Cp}$ button, the reference light curve is determined and subtracted from the light curve of star \#1. The resulting relative (or absolute, if the offset cor. option is used) light curve is displayed in the light curve field. The $>(<)$ button switches to the light curve of the next (previous) star in the coordinate list. The reference light curve is determined without the star currently displayed. PODEX also provides the possibility to exclude stars from calculation of the reference light curve. The stars to be encountered are selected in the use menu. Mean magnitude, variance, D-value, and weight of all light curves are listed in the terminal window. Mean magnitude, standard deviation, and D-value of the currently displayed star are listed in the status line.

\subsection{Outlier rejection}

Outliers can be rejected from the light curve by moving the cursor onto the data point in the light curve field and press the left mouse button. The recently rejected data point can be included again with the undo button.

\section{Output}

With the Save current star button the heliocentric Julian date, relative (or absolute if the offset cor. option is used) magnitude, reference magnitude and the airmass of the actual star are saved in a file. With the Save all stars button the heliocentric Julian date and the instrumental magnitudes of all stars are saved in a single file.

Acknowledgments. This work is supported by the Austrian Fonds zur Förderung der wissenschaftlichen Forschung (FWF) within the project Stellar Atmospheres and Pulsating Stars (P14984), and the Bundesministerium für Verkehr, Innovation und Technologie (BMVIT) via the Austrian Space Agency (ASA). 